\title{
Comparing eclipse observations of the 2008 August 1 solar corona with an MHD model prediction
}

\author{
V. Rušin ${ }^{1}$, M. Druckmüller ${ }^{2}$, P. Aniol ${ }^{3}$, M. Minarovjech ${ }^{1}$, M. Saniga ${ }^{1}$, Z. Mikić ${ }^{4}$, J. A. Linker ${ }^{4}$, \\ R. Lionello ${ }^{4}$, P. Riley ${ }^{4}$, and V. S. Titov ${ }^{4}$ \\ 1 Astronomical Institute, Slovak Academy of Sciences, 05960 Tatranská Lomnica, Slovakia \\ e-mail: vrusin@ta3.sk \\ 2 Faculty of Mechanical Engineering, Brno University of Technology, 61669 Brno, Czech Republic \\ 3 ASTELCO Systems GmbH, Fraunhoferstr. 14, 82152 Martinsried, Germany \\ 4 Predictive Science, Inc., 9990 Mesa Rim Rd., Ste. 170, San Diego, CA 92121, USA
}

Received 29 June 2009 / Accepted 10 February 2010

\section{ABSTRACT}

\begin{abstract}
Context. The structure of the white-light and emission solar coronas and their MHD modelling are the context of our work. Aims. A comparison is made between the structure of the solar corona as observed during the 2008 August 1 total eclipse from Mongolia and that predicted by an MHD model.

Methods. The model has an improved energy formulation, including the effect of coronal heating, conduction of heat parallel to the magnetic field, radiative losses, and acceleration by Alfvén waves.

Results. The white-light corona, which was visible up to 20 solar radii, was of an intermediate type with well-pronounced helmet streamers situated above a chain of prominences at position angles of 48, 130, 241, and 322 degrees. Two polar coronal holes, filled with a plethora of thin polar plumes, were observed. High-quality pictures of the green $(530.3 \mathrm{~nm}, \mathrm{Fe}$ XIV) corona were obtained with the help of two narrow-passband filters (centered at the line itself and the vicinity of $529.1 \mathrm{~nm}$ background), with a $F W H M$ of $0.15 \mathrm{~nm}$.

Conclusions. The large-scale shape of both the white-light and green corona was found to agree well with that predicted by the model. In this paper we describe the morphological properties of the observed corona, and how it compares with that predicted by the model. A more detailed analysis of the quantitative properties of the corona will be addressed in a future publication.
\end{abstract}

Key words. Sun: corona - magnetic fields - magnetohydrodynamics (MHD)

\section{Introduction}

The solar corona is the uppermost layer of the solar atmosphere, whose in-depth scientific understanding is of paramount importance not only for the field of solar physics itself, but - since the extension of the solar corona, in the form of the solar wind, directly influences our Earth - also for geophysics, satellite communications and navigation, aeronautics, massive failures of electrical distribution systems, and biosphere research. Although the corona is much hotter (several hundred times) than the photosphere, its density is only $\sim 10^{-9}$ of the photosphere, and it produces about one-millionth as much visible light. Therefore, the white-light corona can only be observed from the Earth during total eclipses of the Sun and/or by coronagraphs (e.g., at the Mauna Loa Observatory, Hawaii). It is also possible to routinely image the corona with coronagraphs on spacecraft, though the innermost corona (from the solar surface to about 1.5 solar radii) cannot be seen in this way, so eclipses remain an almost unique opportunity to investigate this part of the white-light corona.

It is generally accepted that the structure of the corona is governed by magnetic fields. Apart from large-scale magnetic fields, which vary on a long-term basis and are responsible for the global shape of the corona, there also exist localized small-scale magnetic fields with a high degree of temporal variation, which give rise to a variety of fine coronal structures and phenomena. Although we are still not able to accurately measure weak local coronal magnetic fields, their strength and temporal evolution can indirectly be inferred from those of photospheric magnetic fields. We used a magnetohydrodynamic (MHD) model (Mikić et al. 1999, 2000,2007) to predict the shape of the corona, and to subsequently check the prediction with observations taken during the following eclipse. In this paper we discuss the shape of both the white-light and green emission corona as observed during the August 1, 2008 eclipse by our team, and we compare these with those predicted by the model.

Our focus is to compare the morphology of the observed and modeled corona, and not to make detailed, quantitative comparisons. That will be the subject of a future paper. We do note that these quantitative comparisons are critical to calibrating the (empirical) coronal heating model that is a component of our MHD model (Lionello et al. 2009), because the overall emission from the model as well as its distribution is governed by the amount of heating and the form of the coronal heating function.

\section{The 2008 August 1 total eclipse observations from Mongolia}

Observations of the white-light and green emission corona during the August 1, 2008 total eclipse were carried out by the Czech-German-Slovak expedition based in Mongolia with equipment comprising a number of lenses and telelenses, ranging from $1250 \mathrm{~mm}$ to $200 \mathrm{~mm}$ in focal length. In particular TMB APO $105 \mathrm{~mm}$ with Baader Flat-field FFC EFL, 


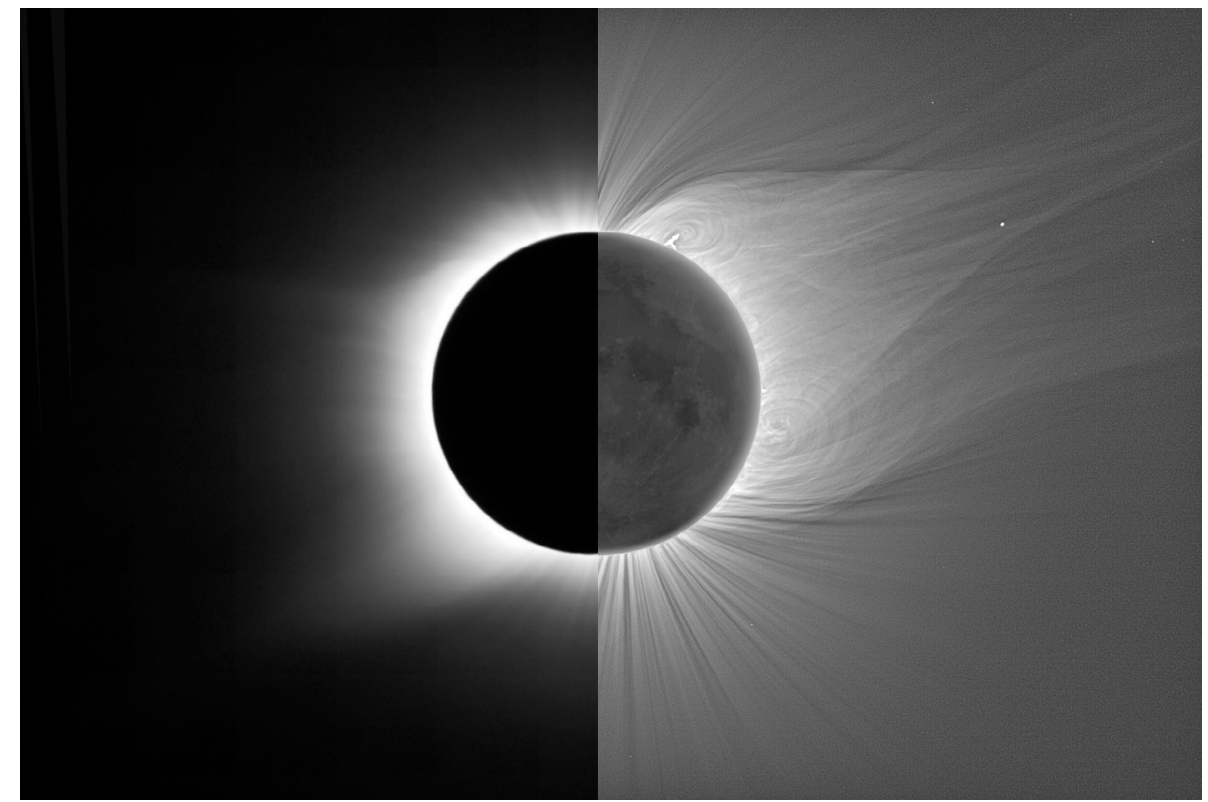

Fig. 1. Shape of the 2008 August 1 eclipse white-light corona in its "raw" form (left) and after digital enhancement (right).

Rubinar 10/1000 mm, Maksutov-Cassegrain 6.3/500 mm, two $5.6 / 400 \mathrm{~mm}$ telelenses and a $2.8 / 200 \mathrm{~mm}$ telelens for the whitelight corona (in the spectral range 400-700 $\mathrm{nm}$ ), and two $500 \mathrm{~mm}, f / 8$ telelenses with $0.3 \mathrm{~nm}$ narrow-passband filters at a working temperature of $45^{\circ} \mathrm{C}$ for the green-line corona observations. One of the filters was centered at the emission spectral line of $530.3 \mathrm{~nm}$, the other at the nearby $529.1 \mathrm{~nm}$ line, both placed in front of the focal plane of the telelenses. This enabled us to subtract the contribution of the white-light corona from the light in $530.3 \mathrm{~nm}$, and thereby get an image of the green-line emission corona. The exposure times were within the interval from $8 \mathrm{~s}$ to $1 / 4000$ of a second. An H-alpha filter with a transition FWHM of $6 \mathrm{~nm}$ and an enhanced sensitivity in the red spectral band was placed in front of the focal plane of one of the two $400 \mathrm{~mm}$ telelenses. Pictures of both the white-light and green emission corona for a total of 255 were taken by the digital cameras CANON EOS 1D MarkIII, 1Ds MarkIII, 5D, 20D and 350D, guided and controlled by special software installed in notebook computers. All the objectives were placed on the same mounting, powered by a Toyota Landrover battery. Thermoregulation of the filters was controlled by a portable $200 \mathrm{~V} / 50 \mathrm{~Hz}$ accumulator.

Our observing site, Bor Udzuur, Mongolia, was located at an altitude of 1223 metres above sea level, about $23 \mathrm{~km}$ south-west of the small village of Altaj. Its coordinates were $N=45^{\circ} 23.251^{\prime}$ and $E=92^{\circ} 06.837^{\prime}$, almost at the central line of the eclipse (umbral depth 97.6\%). The period of eclipse was 18:03:35-18:05:39 local time (i.e., 11:03:35-11:05:39UT), with an observed duration of 2:04 being almost identical to its predicted value (Espenak \& Anderson 2007). During the eclipse the Sun was 22 degrees above the horizon, and observing conditions were excellent. Figure 1 compares the white-light corona, using a logarithmic brightness scale, as composed from 25 individual images of varying exposure (left), with a digitally processed version in order to enhance low-contrast structures invisible to the human eye (right). A novel method (Druckmüller et al. 2006; Druckmüller 2009) was used to process the data obtained from these coordinated observations, giving us images of the white-light corona with high-quality resolution for groundbased observations (see also Pasachoff et al. 2007, for the 2006 eclipse). Although the overall resolution is a few seconds of arc,

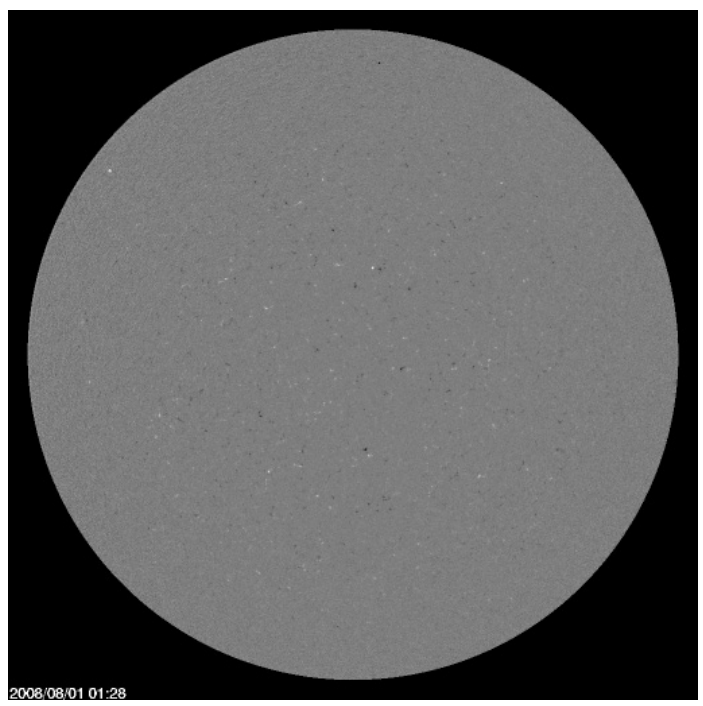

Fig. 2. Magnetogram of SOHO MDI for the eclipse day.

the finest visible features (e.g., polar rays) have a size of around $1 \operatorname{arcsec}(700 \mathrm{~km})$, thanks to the APO $105 \mathrm{~mm}$ lens, whose focal length was increased up to $1250 \mathrm{~mm}$.

\section{The white-light corona}

As evident from Fig. 2, on the day of the eclipse (and for many days around this date) the Sun's photosphere was completely devoid of sunspots, with weak magnetic fields distributed sporadically across the solar disk. Processing and analysis of the white-light coronal images was done separately for three distinct domains, differing from each other by increasing distance from the center of the Sun, to reveal extremely thin, small-scale structures of the inner corona and the pronounced, large-scale features at the most remote, yet still-detectable outer parts of the corona. This strategy was dictated by the fall-off of the temperature and density of the corona with increasing distance from the Sun (e.g. Golub \& Pasachoff 1997) and technical capabilities 

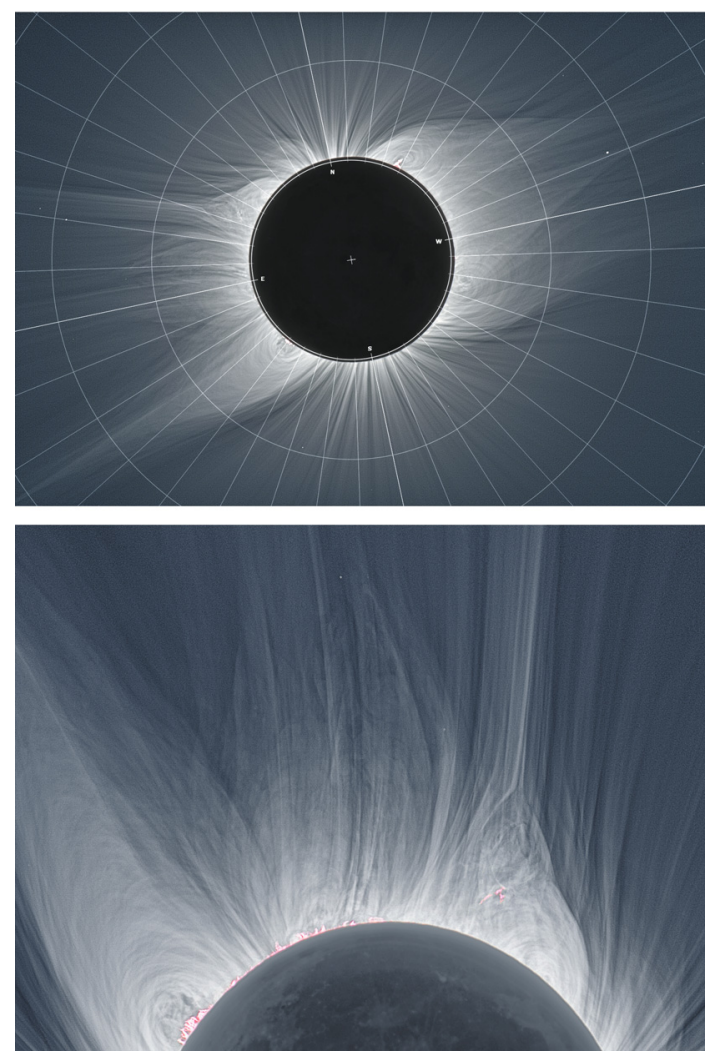

Fig. 3. Overall shape (top) and extra-fine structure over the eastern solar limb (bottom) of the 2008 August 1 eclipse white-light corona. The top figure is oriented so that the celestial pole is vertically up, and the solar limb is indicated by a white circle. The position of the solar north pole is also indicated. In the bottom figure the solar north pole points to the right.

and limitations of the observing equipment employed. A preliminary analysis shows that this eclipse corona is of an intermediate type (Fig. 3) with well-developed polar plumes filling coronal holes around both poles. The northern plumes extended between position angles (PA) of 28 and 340 degrees, the southern ones between 152 and 210 degrees (where the PA is measured counterclockwise from the solar $\mathrm{N}$ pole). The bases of four classical helmet streamers are located over prominence channels at PA 48, 130,241 , and 322 degrees, which is quite typical for the onset of a new solar cycle. H-alpha prominences with a surrounding cavity and dark and bright loops above them were found inside the streamer bases at 130 and 322 degrees. The helmet streamer located at 241 degrees features symmetrical dark and bright loops, whose traces can be seen up to nearly two solar radii, but no $\mathrm{H}$-alpha prominence is visible at its base. On the contrary, a small prominence is seen at the foot of a loop at 258 degrees; yet no loops are visible surrounding it. Also, at 55 degrees and about $150000 \mathrm{~km}$ above the surface we see the beginnings of a prominence. An outline of a huge helmet streamer, anchored between 221 and 339 degrees, is seen in the best pictures. A long chain of small prominences is seen at around 100 degrees. Largescale structures related to helmet streamers can be traced up to distances of 20 solar radii. Associated with the coronal holes is a strange "curtain-like" structure, which we observed previously in the 2006 eclipse corona (Pasachoff et al. 2007).

The Ludendorf index of ellipticity (Ludendorf 1928), as inferred from Fig. 4, amounts to 0.29. This is a typical value characterizing the shape of the corona around minima of solar cycles, although the eclipse corona is, as already mentioned, rather of an

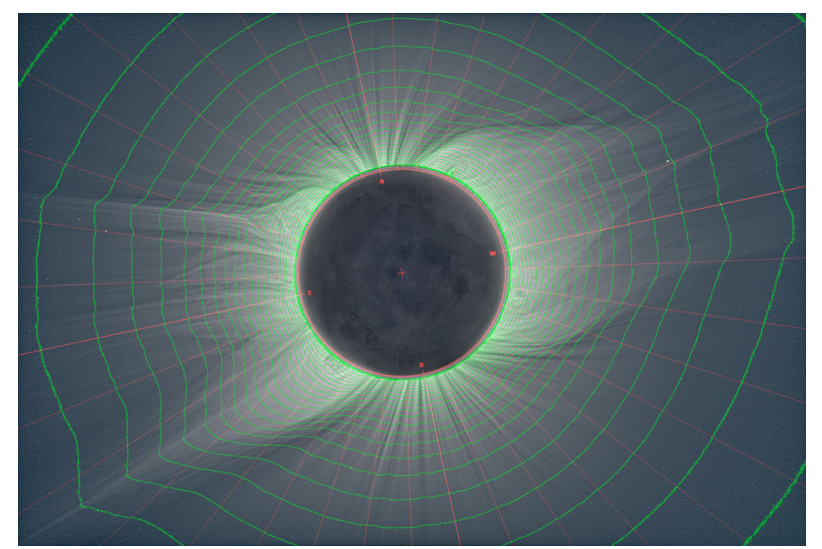

Fig. 4. Isophotes (arbitrary units) based on a logarithmic scale. If the outermost isophote is given the value 1 , the subsequent contour is twice as bright, the next one is four times brighter, etc. These isophotes were used to compute an ellipticity. The solar limb is indicated by a red circle.

intermediate type. Yet, it may well be under-estimated, because in the vicinity of both poles the brightness of the F-corona is already comparable to that of the K-corona at around $1.4 R_{\odot}$ (for a comparison, see Koutchmy \& Magnant 1973).

It is apparent that the 2008 August 1 total eclipse whitelight corona possesses a highly complex and intricate small-scale structure, especially above the eastern limb of the Sun. Its largescale shape agrees reasonably well with the one predicted by the MHD model, as discussed subsequently.

\section{The MHD model prediction}

We previously used a magnetohydrodynamic (MHD) model to predict the coronal structure for total solar eclipses (Mikić et al. 1999, 2000, 2007). The solar photospheric magnetic field, which is routinely measured, can be used as a boundary condition in an MHD model to predict how the magnetic field and plasma is distributed in the corona. Since the solar magnetic field does not evolve substantially near the solar minimum, we expect that magnetic fields measured prior to an eclipse will reasonably predict the structure of the solar minimum corona. The resulting coronal solutions for the plasma density and temperature can be used to estimate the shape of the white-light corona, as well as emission in EUV spectral lines and X-rays. Simulated coronal images of the polarized brightness of the white-light corona and emission images can be generated from the model by integrating along the line of sight perpendicular to the plane of the sky. Our MHD model with improved energy transport was first used to predict the structure of the corona for the March 29, 2006 total solar eclipse (Mikić et al. 2007). The model uses a sophisticated energy equation, including parameterized coronal heating (Lionello et al. 2009), conduction of heat parallel to the magnetic field, radiative losses, and acceleration by Alfvén waves, to produce a significantly better estimate of the plasma temperature and density in the corona than our previous polytropic model (Mikić et al. 2000). This model can describe the equilibration between open and closed magnetic field lines in the corona, including the acceleration of the solar wind along open field lines to supersonic speeds, and includes the steep temperature and density gradients that form in the transition region.

The final state of the corona is obtained by relaxing the 3D time-dependent MHD equations to a quasi-steady state (for about 3.4 days of solar time). These complex calculations run on thousands of processors on massively parallel computers. We 
used our spherical 3D code MAS, which integrates the MHD equations with semi-implicit (Alfvén and sound waves), fully implicit (diffusive terms), and explicit (flow terms) schemes. We solve the very complex sparse matrix equations generated by these algorithms with a preconditioned iterative conjugate gradient solver. Our code is written in Fortran 90 and uses the Message Passing Interface (MPI) for interprocessor communication, and scales very well on many high-performance computer systems.

A preliminary prediction was carried out with photospheric synoptic magnetic field maps from the MDI longitudinal magnetogram aboard SOHO for the Carrington rotation 2071, which covered the interval June 9-July 6, 2008. The calculation was started on July 17 and completed on July 21 . The preliminary prediction was posted on the World Wide Web on July 22. On July 23 we updated the photospheric magnetic field to incorporate MDI measurements up to July 22, and we started the calculation for the final prediction, which was completed 3.6 days later on July 27, when we posted the final prediction on the World Wide Web (Mikić et al. 2008). This calculation was our largest ever, using a 3D mesh with 201191432 mesh points (16.5 million cells) in spherical coordinates $(r, \theta, \phi)$. It was run on the new Ranger massively parallel supercomputer at the Texas Advanced Computing Center (TACC), which in June 2008 was the fourth fastest supercomputer in the world. Figure 5 shows the predicted structure of the white-light corona in polarized brightness, along with traces of some coronal magnetic field lines. These images are sized and rotated identically so they can be compared directly with the sharpened eclipse image. The MHD model predicted the locations of the principal streamers reasonably well, though the high-resolution features observed in the corona are not captured in the model.

Figure 6a shows a synoptic map of the radial component of the photospheric magnetic field that was used for the final prediction. It is apparent that the Sun is very quiet during this time period, with only one small active region present (at 178 degrees Carrington longitude). The photospheric magnetic field is mostly due to dispersed active regions. Nevertheless, the coronal solution has a very rich structure, as discussed previously. Figure $6 \mathrm{~b}$ shows a synoptic map of the computed coronal holes. We define coronal holes as regions in which the field lines open to the solar wind. The coronal hole map is obtained by tracing field lines in the final solution. Coronal holes are colored black, and regions in which the magnetic field lines are closed are colored light gray. The neutral line (i.e., the magnetic field polarity inversion line) in the lower corona at a height $r=1.05 R_{\odot}$ is also shown for reference. The neutral line helps to identify large-scale unipolar areas. The positions of the central meridian and the solar limbs are also shown.

Three principal streamers are evident. The large-scale structure of the streamers compares reasonably well with that seen in the eclipse, although it is apparent that the simulated streamers are less inflated than the observed ones. This is believed to be due to the neglect of sheared (energized) fields in the simulation, which is especially important in the filament channels that accompany the observed prominences described above. It is also evident that the simulated streamer on the west limb does not have as large an extent as the observed streamer. In addition, the high-latitude streamer observed on the NW limb is largely absent in the simulation. This may be due to two reasons. First, the filtering of polar magnetic fields that was performed to generate the synoptic map for the simulation may have affected the accuracy of the field there. This filtering is typically used to reduce errors in estimating the radial component of the magnetic field from
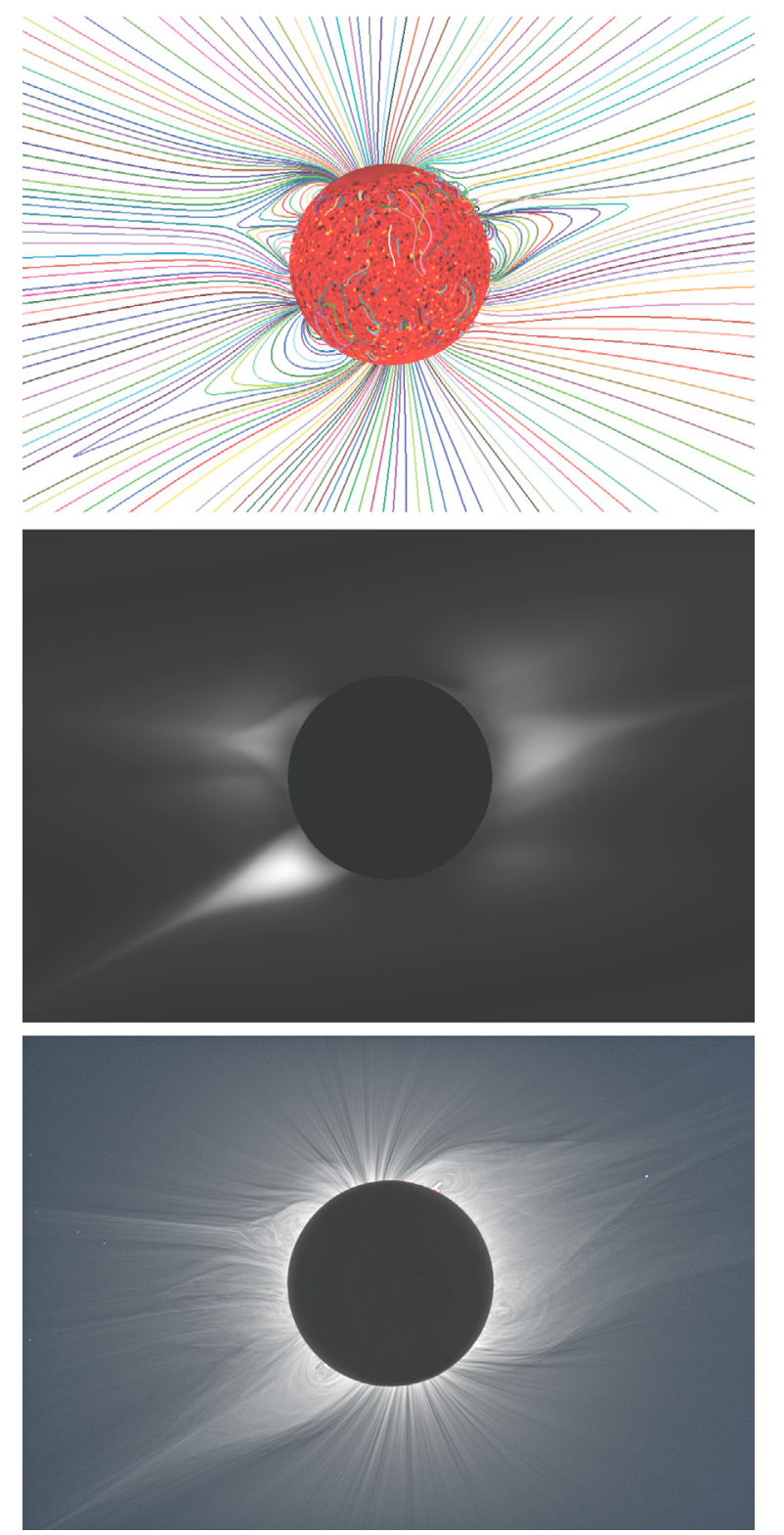

Fig. 5. Comparison between the predicted solar magnetic field lines (top panel) and corona polarization brightness (middle panel) for the 2008 August 1 total solar eclipse from the MHD model and the sharpened white-light eclipse image (bottom panel) from Bor Udzuur, Mongolia. The images are oriented with solar north 12.1 degrees counterclockwise from the vertical direction. It is evident that the bright features in the white-light corona correspond to regions with closed magnetic field lines.

line-of-sight measurements of the field obtained from longitudinal magnetograms. In retrospect we probably should have used less filtering in the polar regions. Second, we may have used a heating specification that opened up too much of the magnetic flux (discussed additionally below). In particular, it can be seen in Fig. 5 that the simulation has largely open magnetic fields in the region of the observed NW streamer. Careful inspection of our simulated coronal hole map Fig. $6 \mathrm{~b}$ indicates that a different coronal heating specification may not have opened as many field lines in that neighborhood. This discussion illustrates that it can be quite an intricate effort to understand the reasons for discrepancies between global coronal models and observations, because discrepancies can arise from inaccuracies in magnetic 

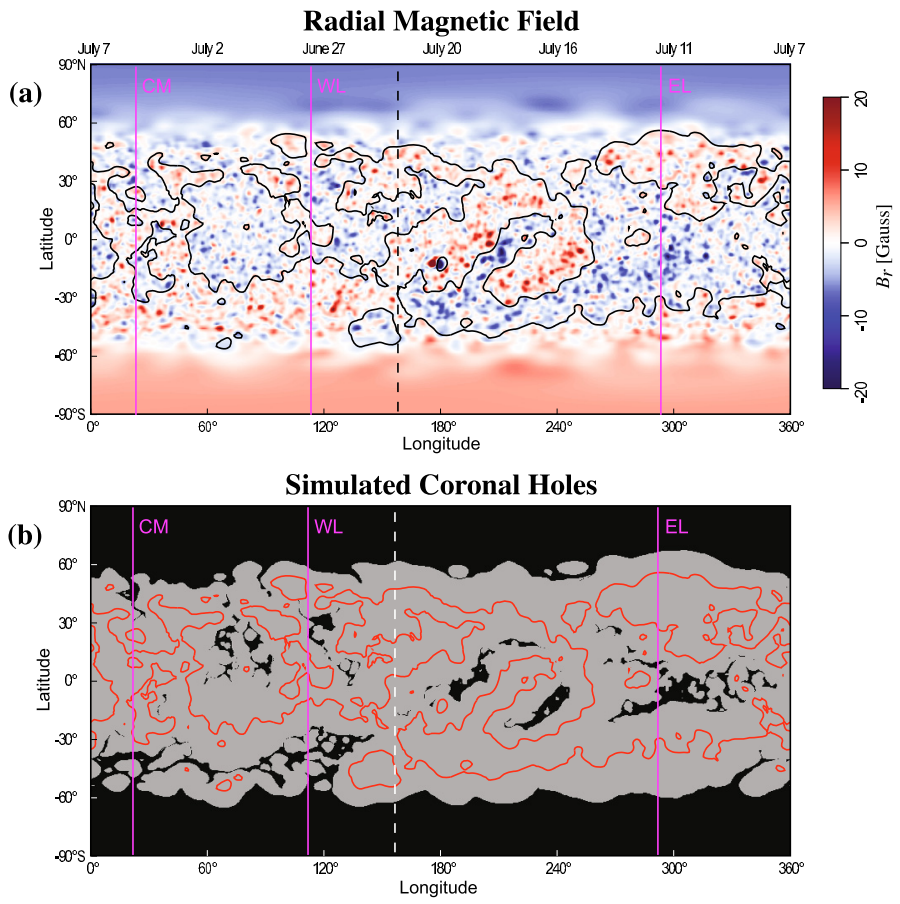

Fig. 6. a) Synoptic map of the radial component of the photospheric magnetic field that was used for the final prediction. The magenta lines indicate the position of the central meridian (CM), east limb (EL), and west limb (WL) at the moment of eclipse. The dates at which the magnetic field was measured at central meridian are shown at the top of the image. The vertical dashed line shows the location at which oldest data (on June 25) and newest data (on July 22) meet. b) A synoptic map of the computed coronal holes, shown in black, and closed field regions, shown in light gray. The neutral line (magnetic polarity inversion line) at $r=1.05 R_{\odot}$ is shown (in black in the top image, and in red in the bottom image) in both panels for reference.

fields or parameters in the plasma model (such as those that control coronal heating). It should be noted from Figs. 5 and 6 that the NE streamer centered at position angle 55 degrees can be identified as a "pseudostreamer", because it has two neutral lines within it (Wang et al. 2007a). The 2006 eclipse also had a pseudostreamer, but on the SW limb (Wang et al. 2007b). The finescale features observed in the (sharpened) eclipse image are also not very emphasized in the simulation, although the simulated magnetic field does contain fine-scale features (see below). It is important to note that the observed eclipse images have been deliberately digitally processed to emphasize small-scale features. In fact, the appearance of the eclipse corona to the eye is very different from that captured in images like that shown in Fig. 3. Nevertheless, it is likely that the limited spatial resolution and the approximations used in the model suppress fine-scale features in the simulated corona. In Fig. 7 we show for the first time an image of the squashing factor $Q$ that describes the topological structure of the coronal magnetic field (Titov \& Démoulin 1999; Titov et al. 2002; Titov 2007). It is obtained by mapping tens of millions of magnetic field lines. High values of $Q$ indicate the presence of separatrices and quasi-separatrix layers, and are believed to be likely sites for the formation of current sheets and concentration of electric current. These are expected to dissipate and lead to localized flows and heating, producing smallscale structure in the corona. The limited spatial resolution in our global simulation does not permit us to explicitly include these small-scale effects. However, we speculate that the rich magnetic field topology shown in Fig. 7 forms a "background state",

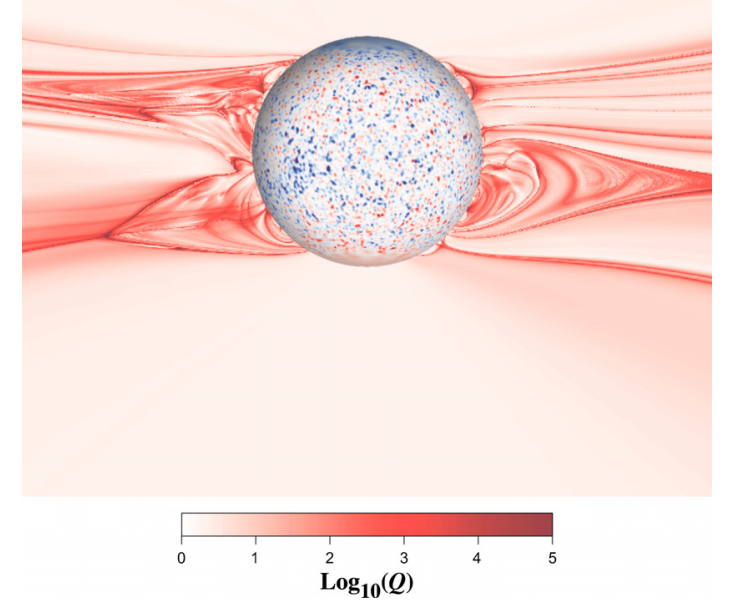

Fig. 7. Mapping of the squashing factor $Q$ in the corona (shown in red using a logarithmic scaling) in the plane of the solar limbs. High values of $Q$ show areas at which intense electric currents are expected to be induced by perturbations driven by small-scale sub-photospheric convective motions. The solar surface is colored according to the radial magnetic field. The tilt due to the solar Bo angle is not included; solar $\mathrm{N}$ is vertically up. We speculate that the dissipation and dynamics of these currents may possibly lead to the formation of fine-scale coronal structure, similar to that seen in the (sharpened) eclipse images.

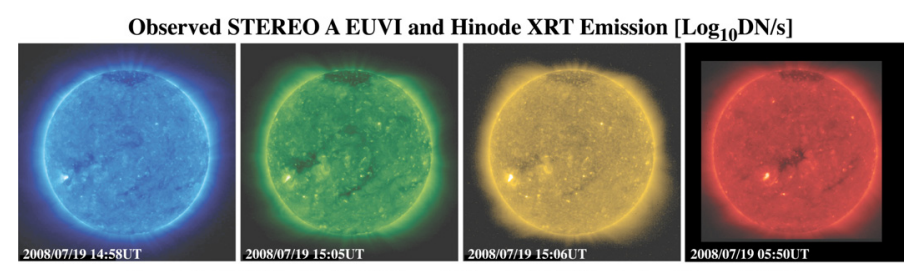

Simulated STEREO A EUVI and Hinode XRT Emission $\left[\log _{10}\right.$ DN/s]

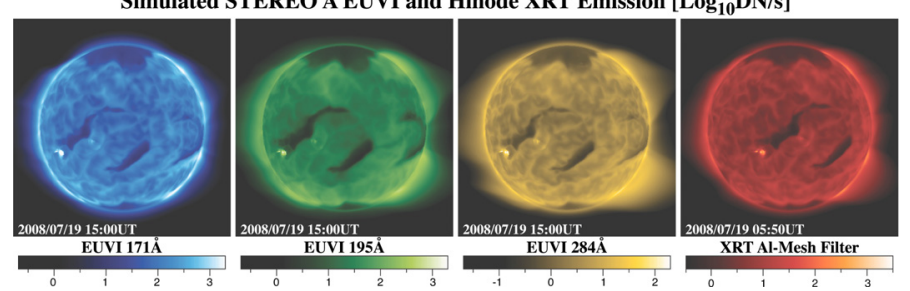

Fig. 8. Comparison between the observed (top panels) and simulated from a 3D MHD model (bottom panels) emission in EUV and X-rays on July 19, 2008. The EUV emission is from the EUVI instrument of the SECCHI Consortium on the STEREO A spacecraft. The X-ray emission is from the XRT instrument on the Hinode spacecraft, and was taken with the $\mathrm{Al}$-mesh filter.

in which these extra physical effects could produce fine-scale structure in the corona, as observed in the processed eclipse images shown in Fig. 3.

In order to demonstrate the emission properties of the corona, we show in Fig. 8 a comparison between the simulated and observed emission in EUV and soft X-rays. We chose to compare these to the picture of July 19, when two equatorial coronal holes appeared on the solar disk, rather than to the picture of August 1, when there were no easily discernible equatorial coronal holes. Our simulated emission in the EUV Fe lines at $17.1 \mathrm{~nm}, 19.5 \mathrm{~nm}$, and $28.4 \mathrm{~nm}$ is compared to STEREO EUVI 

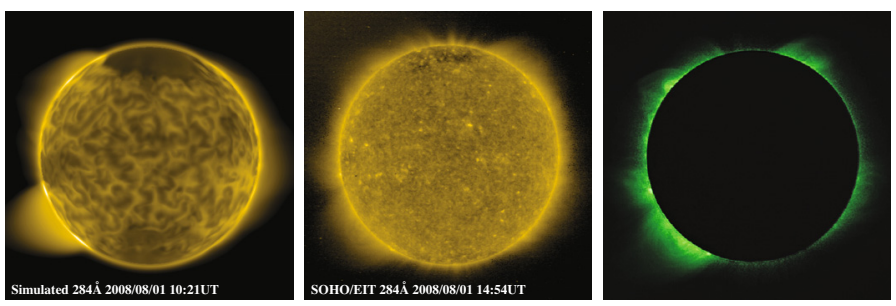

Fig. 9. Shape of the emission corona: predicted emission in the EUV $28.4 \mathrm{~nm}$ line (left) and that observed by SOHO/EIT (middle), compared with the observed green-line eclipse corona (right).

images from the Ahead spacecraft ("STEREO A"), and our simulated X-ray emission is compared to an image from the XRT instrument on Hinode in the Al-mesh filter. It is apparent that our equatorial coronal holes as well as our polar coronal holes are somewhat larger than the observed holes, even if they appear in approximately the correct locations. A detailed comparison between the coronal holes in the simulated and observed emission (not shown) for the whole solar rotation surrounding the eclipse time confirms this conclusion, namely, that the modeled coronal holes are located in approximately the correct places, but tend to be larger than the observed ones.

This is likely indicative of a weakness in our coronal heating specification. (Our polar coronal holes may also be larger than the observed holes due to the smoothing of the polar fields, as discussed earlier.) In the future we will use comparisons like this to improve our coronal heating specification. In this figure we are comparing quantitative estimates of the emission, which demonstrates that our coronal heating has approximately the correct overall size, even though its detailed distribution may need to be improved.

Figure 8 shows that the STEREO view of the Sun (i.e., EUV emission) is perceptibly different from that seen by Hinode (i.e., X-ray emission) due to the significant separation between STEREO A and the Earth at this time (approximately 33 degrees). In the comparison between our simulated emission and observations we take into account the proper location of these spacecraft.

\section{The green-line and $28.4 \mathrm{~nm}$ EUV corona}

While the white-light corona is essentially the light of the photosphere scattered on free electrons and dust particles in the vicinity of the Sun and is quite easy to observe, the radiation of the emission corona is associated with specific spectral lines (e.g. Billings 1966). It is the inherent radiation of the corona by highly ionized elements of iron, calcium, nickel, etc. A great majority of the corresponding lines lie in the extreme UV or X-ray part of the spectrum; in the visible region there are only about 28 lines, the brightest of them being Fe XIV at $530.3 \mathrm{~nm}$, whose half-width is about $0.1 \mathrm{~nm}$. Accordingly, while making an image of the white-light corona of sufficient quality is technically not too demanding a task, while that of the emission corona - for example, in the Fe XIV line, which is characteristic for the parts of the corona with a temperature of about 1.8 million $\mathrm{K}$ - requires the use of a narrow-passband filter. The use of such filters requires prolonged exposure times, and there are usually difficulties with separation of the line from the continuum. As far as we know, all observations of the $530.3 \mathrm{~nm}$ emission corona (also known as the green-light corona) have previously been based on the use of a single filter centered at the line in question (e.g. Badalyan \& Sýkora 1997). Moreover, it was assumed that the

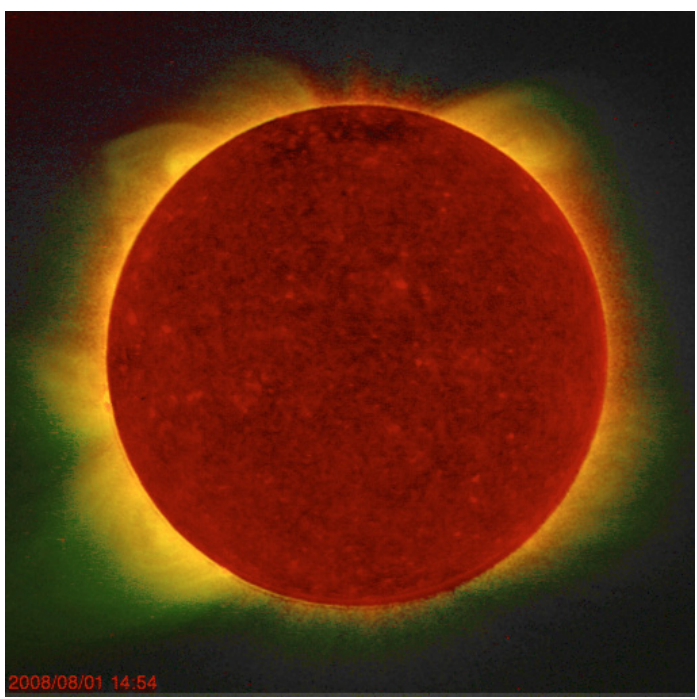

Fig. 10. Superposition of images of the $28.4 \mathrm{~nm}$ and $530.3 \mathrm{~nm}$ corona on eclipse day. The yellow color represents those regions in the corona, where both intensities are increased. The green color: the green corona has higher intensities than the $28.4 \mathrm{~nm}$ corona, the red color is an opposite state. (The $28.4 \mathrm{~nm}$ corona: ESA/NASA/SOHO/EIT.)

contribution from the white-light corona, which had to be subtracted, is the same everywhere. This is a poor assumption, because the radial gradient in the intensity of the white light-corona is very steep near the solar limb (Koutchmy 1997), so that the resulting images of the green-light corona were always considerably distorted by the radiation of the white-light corona. In order to overcome this difficulty, we employed two telelenses with two narrow-passband filters, with a $F W H M$ of $0.15 \mathrm{~nm}$ each; one filter was centered at the Fe XIV line itself, the other at the continuum around $529.1 \mathrm{~nm}$. The pictures of both "coronae" were taken simultaneously. This way we succeeded in obtaining highresolution images of the virtually "pure" green-light corona; one such image is shown in Fig. 9 (right). This is a new technique that differs from the classical ones used in the early 1970s based on the use of a single filter by tilting and accurately calibrating it (e.g. Fort et al. 1972; Picat et al. 1979).

The predicted shape of the emission corona made by Mikić et al. (2008) was generated for the following SOHO/EIT EUV lines: $17.1 \mathrm{~nm}$ (Fe IX/X, formed at temperatures around $1 \mathrm{MK})$, $19.5 \mathrm{~nm}$ (Fe XII, 1.5 MK), and 28.4 nm (Fe XV, $2 \mathrm{MK}$ ), as well as for soft $\mathrm{X}$ rays (more than $2 \mathrm{MK}$ ). We therefore compare our green-line coronal image with the closest temperature EUV line, namely the $28.4 \mathrm{~nm}$ EUV prediction, as shown in Fig. 9. We also show the $28.4 \mathrm{~nm}$ observation from SOHO/EIT closest to the time of the eclipse. The agreement as seen in Fig. 10, in which we superimpose the green-line and $28.4 \mathrm{~nm}$ images, is reasonably good, though there are small differences in the detailed emission within the streamers, as already noted in Sect. 4. This agreement suggests that the long-term but infrequent observations of the green corona with ground-based coronagraphs could be complemented by the rich database of recent $28.4 \mathrm{~nm}$ coronal observations from SOHO/EIT to create a coronal index of solar activity (Pasachoff et al. 2006).

\section{Summary and conclusion}

We made a comparison between the structure of both the white-light and emission solar corona based on the data obtained during the total eclipse of August 1, 2008, by the 
Czech-Slovak-German expedition from Altaj (Mongolia) and that predicted by an MHD model (Mikić et al. 2008). Our analysis shows that the August 1, 2008 eclipse white-light corona possesses a highly complex and intricate structure (helmet streamers, polar plumes, coronal holes, narrow radially oriented or slightly curved streamers, loops), especially above the eastern limb of the Sun. Its large-scale shape agrees well with the one predicted, except for its fine-scale structure, which is beyond the capabilities of the model. A careful inspection of the original images reveals a large number of super-fine features that are about or slightly less than 1 arcsec across, i.e., about $700 \mathrm{~km}$. Such fine spatial resolution has so far been reported only by Nesmyanovich et al. (1974) from observations of the eclipse in 1968 by a telescope, whose focal length was $10 \mathrm{~m}$, and by Vsekhsvjatsky et al. (1970) and November \& Koutchmy (1996), who went even below 1 arcsec thanks to excellent observing conditions and the use of a powerful $3.6 \mathrm{~m}$ Canada-France-Hawaii telescope in Hawai (see also Koutchmy \& Stellmacher 1976; and Koutchmy et al. 1994). Apart from well-known loops at the bases of helmet streamers and radial or slightly bent rays and plumes, our pictures also reveal a number of bright and dark structures of a helical shape, which to the best of our knowledge are reported for the first time.

The green line corona was less well pronounced, which is typical for periods around the solar minimum (see also the very recent paper by Habbal et al. 2010). The EUV corona was faint as well, and was most conspicuous for the eastern limb of the Sun and regions close to the equator. The $28.4 \mathrm{~nm}$ corona, as observed from $\mathrm{SOHO}$, was visible across the whole solar disk, with the exception of coronal hole regions. Its shape is similar to that of the green corona, whose maximum was observed at the foot of the helmet streamer at 132 degrees. We also note that the span of the coronal hole above the north pole was less than predicted. The particular distribution of helmet streamers seems to indicate that a new solar cycle has already begun, despite only a very sporadic occurrence of sunspots and the fact that the year 2009 was also very quiet.

Acknowledgements. This work (Rušin, Saniga, Minarovjech) was partially supported by the Slovak Academy of Sciences Grant Agency VEGA, Grant 0098, and by the Science and Technology Assistance Agency APVT under contract APVT 51-012-704. Druckmüller's research was partially supported from the Ministry of Education of the Czech Republic, research plan MSM0021630518 and by grant 205/09/1469 of Czech Science Foundation. Aniol acknowledges the support received from ASTELCO. The group at Predictive Science is supported by NASA's Heliophysics Theory, Living With a Star, and SR\& T Programs, by the Center for Integrated Space Weather Modeling (an NSF Science \& Technology Center), and by AFOSR's Space Sciences program. We thank the staff at the Texas Advanced Computing Center (TACC) for graciously providing us with dedicated time on their supercomputer Ranger, and NASA's Advanced Supercomputing Divison (NAS) for an allocation on the Columbia supercomputer. We thank Yang Liu and Todd Hoeksema of Stanford University for providing us with timely access to MDI magnetograph data. Our special thanks go to Prof. Batmunch of the Center for Astrophysics and Geophysics, Ulaan Baattar, for his kind guidance, help and assistance at various stages of the expedition. Finally, we are grateful to the other members of our team - M. Ditzer, L. Klocok, K. Martišek, J. Sladeček, and P. Zimmermann - who substantially contributed to the success of the expedition.

\section{References}

Billings, D. E. 1966, A guide to the solar corona (New York and London: Acad. Press)

Badalyan, O. G., \& Sýkora, J. 1997, A\&A, 319, 664

Espenak, F., \& Anderson, J. 2007, Total solar eclipse of 2008 August 01, NASA/TP-2007-214149

Druckmüller, M. 2009, ApJ, 706, 1605

Druckmüller, M., Rušin, V., \& Minarovjech, M. 2006, Contrib. Astron. Obs. Skalnaté Pleso, 36, 131

Fort, B., Picat, J. P., Combes, M., \& Felenbok, P. 1972, A\&A, 17, 55

Golub, L., \& Pasachoff, J. M. 1997, The Solar Corona (Cambridge, UK: Cambridge University Press)

Habbal, S. R., Druckmüller, M., Morgan, H., et al. 2010, ApJ, 708, 1650

Koutchmy, S. 1997, in Theoretical and Observational Problems Related to Solar eclipse, ed. Z. Mouradian, \& M. Stavinschi, NATO ASI Ser., 494, 39

Koutchmy, S., \& Magnant, F. 1973, ApJ, 186, 671

Koutchmy, S., \& Stellmacher, G. 1976, Sol. Phys., 49, 253

Koutchmy, S., Belmahdi, M., Coulter, R. L., et al. 1994, A\&A, 281, 249

Lionello, R., Linker, J. A., \& Mikić, Z. 2009, ApJ, 690, 902

Ludendorf, H. 1928, Sitz. Ber. Preuss. Akad. Berlin, 16, 185

Mikić, Z., Linker, J. A., Schnack, D. D., et al. 1999, Phys. Plasmas, 6, 2217

Mikić, Z., Linker, J. A., Riley, P., \& Lionello, R. 2000, in The Last Total Solar Eclipse of the Millennium, ed. W. Livingston, \& A. Özgüç (San Francisco, CA: ASP), ASP Conf. Ser., 205, 162

Mikić, Z., Linker, J. A., Lionello, R., et al. 2007, in Solar and Stellar Physics Through Eclipses, ed. O. Demircan, S. O. Selam, \& B. Albayrak (San Francisco, CA: ASP), ASP Conf. Ser., 370, 229

Mikić, Z., Linker, J. A., et al. 2008, Predicting the Structure of the Solar Corona During the August 1, 2008 Total Solar Eclipse, posted to the web site http://wWw . predsci.com/corona/aug08eclipse/

Nesmyanovich, A. T., Dzjubenko, N. I., Khomenko, J.A, \& Popov, O. S. 1974, Astron. Zh., 51, 517

November, L., \& Koutchmy, S. 1996, ApJ, 466, 512

Pasachoff, J. M., Kimmel, S. B., Druckmüller, M., Rušin, V., \& Saniga, M. 2006, Sol. Phys., 238, 261

Pasachoff, J. M., Rušin, V., Druckmüller, M., \& Saniga, M. 2007, ApJ, 665, 824

Picat, J. P., Felenbok, P., \& Fort, B. 1979, A\&A, 75, 176

Titov, V. S. 2007, ApJ, 660, 863

Titov, V. S., \& Démoulin, 1999, A\&A, 351, 707

Titov, V. S., Hornig, G., \& Démoulin, P. 2002, J. Geophys. Res., 107, 1164

Vsekhsvjatsky, S. K., Dzjubenko, N. I., Ivanchuk, V. I., \& Rubo, G. A. 1970, Solnechnyje Dannyje, 9, 88

Wang, Y.-M., Sheeley, N. R., Jr., \& Rich, N. B. 2007a, ApJ, 658, 1340

Wang, Y.-M., Biersteker, J. B., \& Sheeley, N. R., et al. 2007b, ApJ, 660, 882 